\title{
The Relationship among Leisure Involvement, Leisure Benefits, and Happiness of Elementary Schoolteachers in Tainan County
}

\author{
Chao-Chien Chen \\ Department of Leisure and Recreation Management, Asia University \\ 500, Lioufeng Rd., Wufeng, Taichung 41354, Taiwan \\ Tel: 886-4-23323456. E-mail: peter72@asia.edu.tw
}

Chia-Hsin Cheng (Corresponding author)

Department of Management, National Yunlin University of Science and Technology

No.123, University Rd, Section 3, Douliou, Yunlin 64002, Taiwan

Tel: 886-87212372. Fax: 886-8-7625440. E-mail: g9720801@yuntech.edu.tw

\begin{abstract}
Shih-Yen Lin
Department of Leisure and Tourism Management, National Chi Nan University

No, 1, University Rd., Puli, Nanton County 54561, Taiwan
\end{abstract}

Tel: 886-492-910-960. E-mail: sylin5016@ncnu.edu.tw

Received: May 9, 2013 Accepted: June 7, 2013 Published: July 8, 2013

doi:10.5296/ire.v1i1.3970 URL: http://dx.doi.org/10.5296/ire.v1i1.3970

\begin{abstract}
We examined the relationships among leisure involvement, leisure benefits, and happiness from the perspective of elementary schoolteachers. The results of independent sample $t$-test show that the male teachers in participating in leisure activities reaches a significant difference compared to female teachers including leisure involvement, leisure benefits and happiness. From the one-way ANOVA results indicated that the elementary schoolteachers
\end{abstract}


have significant differences in ages and job position variables for leisure involvement and in teaching years and job position for leisure benefits. Finally, the elementary schoolteachers have significant differences in education, teaching years, and job position for happiness. Leisure involvement and leisure benefits most strongly predict the happiness of elementary schoolteachers.

Keywords: leisure involvement, leisure benefits, happiness, job position

\section{Introduction}

Why is leisure important? And why should a strategically planned leisure activities program be developed and implemented in Taiwan public schools? Recently, the work of education reform has actively promoted and required that teachers be more specialized, diversified and technological (Tsai, 2004). This has placed heavy job pressure and loading on teachers and reduced leisure and relaxation. Related studies have indicated that participating in sports and leisure activities are effective methods for relieving pressure, suggesting that teachers should actively participate in leisure activities to promote health, happiness, and social relationships, and to attain a sense of accomplishment that enhances teaching efficiency (Chen, 2004; Chang et al., 2001).

Participating in leisure activities relieves people of work fatigue, monotony, and work pressures, and attains self-realization from work disruption (Damanedier, 1974). Shiver (1981) indicated that whether in schools, the workplace, family, or community, leisure activities play a leverage role in life and establish a process whereby people develop skills, interests, and competence that enhance and improve their lives (Dattilo \& Murphy, 1991). Leisure is a self-development tool that includes physical, mental, emotional, spiritual, and social benefits (Pichly, 2002)

Participation refers to commitment and involvement, and may be appropriately represented by the concept of enduring involvement (McIntyre, 1989). Rothschild (1984) defined involvement as a psychological state of motivation, arousal, or interest between a person and an activity or product that is driven by current external variables (the situation; the product; the communications) and past internal variables (enduring; ego; central values). Involvement is the level of perceived personal importance evoked by a stimulus (or stimuli) within a specific situation (Huang \& Chen, 2005), conceptualized as an attitude that a person develops or learns through interaction with the social environment (Sherif et al., 1965).

Leisure researchers have more widely used the term involvement (Havitz et al., 1993; McIntyre, 1989; Schuett, 1993) and a certain consensus has developed on these issues including attraction, self-expression and centrality (Mclntyre \& Pigram, 1992). Attraction is a relatively obvious or intuitive component of involvement in recreational activities that should be conceptualized as a combination of importance and pleasure. Self-expression is similar to the sign, symbol, or self-impression that people wish to convey to others through their leisure participation or choice of consumer product. The centrality of leisure or of a particular leisure activity includes a person's perception that the activity has valued life benefits, such as pressure reduction or other significant health outcomes. The three facets of attraction, 
self-expression, and centrality represent conceptually separate and distinct aspects of leisure involvement (Havitz \& Dimanche, 1997).

People believe that leisure involvement leads to a sense of pleasure and feelings (Godbey, 1980) that cause physical, economic, environmental, social, and psychological effects according to personal evaluation, which produces leisure benefits. In other words, leisure benefits resulting from participation in leisure activities enhances the personal satisfaction of people's needs and self-achievement (Berger \& Wankel, 1991). Cordes and Ibrahim (1999) asserted that benefits of leisure including physiological benefits, social benefits, psychological benefits, and emotional benefits that can help people to relieve job pressure, bring relaxation and interest to daily life, and balance physical and psychological development.

Happiness has recently become a highly valued topic (Nawijn, 2011). Numerous studies have indicated that leisure benefits enhance personal happiness (Spark et al., 2003; Hallab, 2006) and are also a tool for developing better human beings (Pichly, 2002). Diener (1984) defined happiness as an affectively oriented evaluation of well-being that entails a preponderance of positive effect over negative effect. Happiness involves a degree of life, job, physical, and psychological pleasure. People improve their happiness by changing their actions (Sheldon \& Lyubomirksy, 2006), including investing time in social relationships (Diener \& Seligman, 2002) or actively participating in sports (Hills \& Argyle, 1998) to increase happiness. The leisure domain is an appropriate domain to improve a person's sense of happiness (Ateca-Amestoy et al., 2008; Spiers \& Walker, 2008). Previous empirical research has indicated that pleasant leisure involvement and leisure benefits are significantly positively correlated with happiness (Reich \& Zautra, 1981), a higher degree of happiness (Berger \& McInman, 1993; Lin, 2004), and significant predictors of happiness ( $\mathrm{Lu} \& \mathrm{Hu}, 2005$ ). Therefore, leisure involvement and leisure benefits can promote happiness ( $\mathrm{Lu} \&$ Argyle, 1994; Currier, 2004).

Elementary schoolteachers are basic education instructors and play important roles for elementary students. Therefore, elementary schoolteachers who actively participate in leisure activities and subsequently enhance job satisfaction and teaching efficiency, fulfill self-accomplishment and self-confidence, attain happiness, impart to students an improved understanding of leisure, and help students develop an awareness of the importance of leisure in society and recognize the significant values that it may contribute to their lives and well-being (Kraus, 1994). Thus, our research purposes are as follows:

1) Understand the leisure involvement, leisure benefits, and happiness of elementary schoolteachers in Tainan County.

2) Compare the demographic differences of leisure involvement, leisure benefits, and happiness on participating in leisure activities of elementary schoolteachers in Tainan County.

3) Understand the relationship among the leisure involvement, leisure benefits, and happiness of elementary schoolteachers in Tainan County. 
4) Understand the predictive effectiveness of leisure involvement on leisure benefits and happiness.

Therefore, we present the following hypotheses:

H1: A significant demographic difference exists on the leisure involvement of participating in leisure activities by elementary schoolteachers in Tainan County.

H2: A significant demographic difference exists on the leisure benefits of participating in leisure activities by elementary schoolteachers in Tainan County.

H3: A significant demographic difference exists on happiness in participating in leisure activities by elementary schoolteachers in Tainan County.

H4: A positive relationship exists between leisure involvement and the leisure benefits of elementary schoolteachers in Tainan County.

H5: A positive relationship exists between leisure involvement and the happiness of elementary schoolteachers in Tainan County.

H6: A positive relationship exists between leisure benefits and the happiness of elementary schoolteachers in Tainan County.

H7: Leisure involvement and leisure benefits positively predict the happiness of elementary schoolteachers in Tainan County.

\section{Methods}

\subsection{Participants}

Our sample was drawn from the "Elementary school register of the Tainan County Government Education", including 46 elementary schools and 3,015 teachers in Tainan County. The sample of this study contains two parts: pilot study sample and research participants. First, we use the pilot study to confirm the content validity of the questionnaires, and the pilot study sample was based on purposive sampling of 200 pilot questionnaires from Sie-Jin Elementary School, Andian Elementary School, Simes Elementary School, Cheng Kung Elementary School, Sishu Elementary School, and Sing-gong Elementary School etc... six schools.

Then, a stratified random sampling survey was adopted as the data collection method of this study according to the "Elementary school register of the Tainan County Government Education". The school was divided into small-sized (12 classes), medium-sized (13 to 21 classes), large-sized (25 to 39 classes), and extra-large-sized (more than 40 classes), according to the school size to determine the proportion of each layer and to randomly select each layer of the school number, then, getting the number of samples. Thus, 600 questionnaires were collected; however, 29 questionnaires were incomplete and deleted. The final sample size for statistical analysis was 571.571 divided by 600 and the response rate was $95.2 \%$. 


\subsection{Data Collection Instrument}

The questionnaire consisted of four major parts. The first part assessed leisure involvement. We adopted the leisure involvement questionnaire from leisure involvement scales (McIntyre \& Pigram, 1992), which was slightly modified to include 14 question items. The second part was composed of 13 question items to assess the leisure benefits adapted from the study by Lin (2002), Hong (2002), and Lin (2004), which was modified to measure the leisure benefits of elementary schoolteachers in Tainan County who participate in leisure activities. The third part assessed happiness and was adapted from the Oxford Happiness Inventory Scale (Argyle, 1987), which contains 24 question items designed to assess happiness. Each questionnaire item was measured using a 5-point Likert-type scale, ranging from 1 (strongly disagree) to 5 (strongly agree). The final part detailed respondent backgrounds.

\subsection{Data Analysis}

SPSS 12.0 was used to analysis the questionnaire data according to the research hypotheses, and the analysis methods are as follows:

(1) Descriptive analysis:

To understand the demographic profiles of the respondents and the situation on leisure involvement, leisure benefits and happiness of elementary schoolteachers in Tainan County participate in leisure activities.

(2) Independent Sample $t$-test analysis:

To explore the differences between the genders on leisure involvement, leisure benefits, and happiness of elementary schoolteachers in Tainan County participate in leisure activities.

(3) One-Way ANOVA:

To explore the differences between socio-demographic variables, including age, education degree, teaching years, and job position on leisure involvement, leisure benefits, and happiness of elementary schoolteachers in Tainan County participate in leisure activities.

(4) Correlation analysis:

To explore the relationship among leisure involvement, leisure benefits, and happiness.

(5) Regression analysis:

To analyze the effect of leisure involvement and leisure benefits on happiness.

\section{Result}

\subsection{Item Analysis}

Our study used the internal consistency criterion to test the scale validity. Using the t-test to obtain the critical ratio value that is greater than 3 and that reached the significance level, we used the correlation to calculate each item and the total scale. We chose the correlation coefficient of the total scale greater than 0.3 (Wu \& Tu, 2005), which means those items have 
discriminatory power and can be used as the construct and appropriateness reference.

\subsection{Factor Analysis and Reliability Test}

Table 1. Summary of factor analysis results on leisure involvement $(\mathrm{N}=571)$

\begin{tabular}{|c|c|c|c|}
\hline & & Factor Loadings & \\
\hline Item & Attraction & Centrality of Lifestyle & Self-Expression \\
\hline 3 & .707 & & \\
\hline 2 & .686 & & \\
\hline 4 & .718 & & \\
\hline 5 & .737 & & \\
\hline 1 & .717 & & \\
\hline 7 & & .704 & \\
\hline 8 & & .735 & \\
\hline 6 & & .741 & \\
\hline 9 & & .732 & \\
\hline 10 & & .724 & \\
\hline 11 & & & .711 \\
\hline 12 & & & .738 \\
\hline 14 & & & .679 \\
\hline 13 & & & .636 \\
\hline Cronbach's $\alpha$ & .916 & .940 & .934 \\
\hline Eigenvalues over & 3.620 & 2.239 & 2.002 \\
\hline$\%$ of Variance & 27.288 & 25.996 & 17.158 \\
\hline Comulative \% & 27.288 & 52.284 & 70.442 \\
\hline
\end{tabular}

We used factor analysis to test the scale reliability. The leisure involvement scale was extracted from three factors, according to the item characteristics for each factor, including attraction, centrality of lifestyle, and self-expression. The Cronbach's $\alpha$ value of three leisure involvement factors are .916, .940 and .934 (Table 1).

Table 2 shows the summary factor analysis results on leisure benefits. The leisure benefits scale was extracted from three factors: physiological benefits, psychological benefits, and social benefits. The Cronbach's $\alpha$ value of three leisure benefits factors are .830, .880 and .875 .

Table 3 shows the summary factor analysis results on happiness. The happiness scale was extracted from four factors: life satisfaction, interpersonal communication, assertiveness, and health. The Cronbach's $\alpha$ value of four happiness factors are $.890, .887, .820$, and .826 . 


\section{Macrothink}

Table 2. Summary of factor analysis results on leisure benefits $(\mathrm{N}=571)$

\begin{tabular}{|c|c|c|c|}
\hline \multirow[b]{2}{*}{ Item } & \multicolumn{3}{|c|}{ Factor Loadings } \\
\hline & $\begin{array}{c}\text { Physiological } \\
\text { Benefits }\end{array}$ & Psychology Benefits & Social Benefits \\
\hline 4 & .730 & & \\
\hline 5 & .707 & & \\
\hline 3 & .876 & & \\
\hline 1 & .855 & & \\
\hline 2 & .802 & & \\
\hline 8 & & .835 & \\
\hline 9 & & .799 & \\
\hline 10 & & .828 & \\
\hline 7 & & .793 & \\
\hline 6 & & .770 & \\
\hline 11 & & & .802 \\
\hline 12 & & & .693 \\
\hline 13 & & & .744 \\
\hline Cronbach's $\alpha$ & .830 & .880 & .875 \\
\hline Eigenvalues over & 3.170 & 3.003 & 1.823 \\
\hline$\%$ of Variance & 25.151 & 27.715 & 16.332 \\
\hline Comulative \% & 25.151 & 52.866 & 69.198 \\
\hline
\end{tabular}

All the Cronbach's $\alpha$ values fall within the acceptable range between 0.80 to 0.90 , which means very good (Kaiser, 1974) and have been measured as adequate in meeting the criteria recommended by Nunnally (1978). These values indicate the internal consistency of each factor item.

\subsection{Descriptive Analysis}

\subsubsection{Demographic Analysis of Participants}

Table 4 presents a summary of the demographic profiles of the respondents, consisting of $25.57 \%$ male participants and $74.43 \%$ female participants. More than $45.71 \%$ of the respondents were between the ages of 41 to 50 , and over $97 \%$ of the participants had higher educational degrees. Approximately $68.83 \%$ of respondents had taught more than 10 years, and most respondents were homeroom teachers in elementary schools $(64.45 \%)$. 
3.3.2 Demographic Analysis of Leisure Involvement

Table 3. Summary of factor analysis results on happiness $(\mathrm{N}=571)$

\begin{tabular}{|c|c|c|c|c|}
\hline \multirow[b]{2}{*}{ Item } & \multicolumn{4}{|c|}{ Factor Loading } \\
\hline & Life Satisfaction & Assertiveness & $\begin{array}{c}\text { Interpersonal } \\
\text { Communication }\end{array}$ & Health \\
\hline 4 & .607 & & & \\
\hline 6 & .631 & & & \\
\hline 5 & .647 & & & \\
\hline 2 & .546 & & & \\
\hline 3 & .561 & & & \\
\hline 7 & .719 & & & \\
\hline 1 & .653 & & & \\
\hline 11 & & .546 & & \\
\hline 13 & & .731 & & \\
\hline 8 & & .696 & & \\
\hline 9 & & .503 & & \\
\hline 10 & & .517 & & \\
\hline 12 & & .549 & & \\
\hline 19 & & & .777 & \\
\hline 18 & & & .623 & \\
\hline 17 & & & .503 & \\
\hline 15 & & & .547 & \\
\hline 16 & & & .729 & \\
\hline 14 & & & .655 & \\
\hline 22 & & & & .665 \\
\hline 20 & & & & .617 \\
\hline 21 & & & & .621 \\
\hline 24 & & & & .627 \\
\hline 23 & & & & .646 \\
\hline Cronbach's $\alpha$ & .890 & .887 & .820 & .826 \\
\hline Eigenvalues over & 3.996 & 3.318 & 2.251 & 1.891 \\
\hline$\%$ of Variance & 29.985 & 15.491 & 12.214 & 8.711 \\
\hline Comulative $\%$ & 29.985 & 44.475 & 58.689 & 64.400 \\
\hline
\end{tabular}


Table 4. Demographic profile results of respondents

\begin{tabular}{llcc}
\hline Demographic & Item & No. $(\mathrm{N}=571)$ & $\%$ \\
\hline \multirow{2}{*}{ Sex } & Male & 146 & 25.57 \\
& Female & 425 & 74.43 \\
\hline \multirow{4}{*}{ Age } & Below 30 years & 79 & 13.84 \\
& 31-40 years & 197 & 34.50 \\
& 41-50 years & 261 & 45.71 \\
& 51-60 years & 34 & 5.95 \\
\hline \multirow{3}{*}{ Education Degree } & College & 11 & 1.93 \\
& University & 384 & 67.25 \\
& Above Master's & 176 & 30.82 \\
\hline \multirow{3}{*}{ Teaching Years } & Below 10 years & 178 & 31.17 \\
& 11-20 years & 226 & 39.58 \\
& 21-30 years & 158 & 27.67 \\
& Above 31 years & 9 & 1.58 \\
\hline \multirow{3}{*}{ Job Position } & Director Teacher & 33 & 5.78 \\
& Administrative Teacher & 95 & 16.64 \\
& Homeroom Teacher & 368 & 64.45 \\
& Subject Teacher & 75 & 13.13 \\
\hline \multirow{3}{*}{} & & &
\end{tabular}

Table 5. Demographic result on leisure involvement

\begin{tabular}{lcc}
\hline & Mean & Std. Deviation \\
\hline Leisure Involvement & 4.086 & 7.200 \\
Attraction & 4.566 & 2.441 \\
Centrality of Lifestyle & 3.806 & 3.692 \\
Self-Expression & 3.837 & 2.552 \\
\hline
\end{tabular}

Table 5 shows the demographic analysis results of leisure involvement $(\mathrm{M}=4.086)$, indicating that elementary schoolteachers have a high degree of leisure involvement in participating in leisure activities; the attraction factor $(\mathrm{M}=4.566)$ is the highest; the centrality of lifestyle factor is the lowest score $(M=3.806)$. Elementary schoolteachers in Tainan County primarily participate in leisure activities because of the attraction factor.

\subsubsection{Demographic Analysis of Leisure Benefits}

Table 6 shows the demographic analysis result of leisure benefits $(M=3.887)$, indicating that elementary schoolteachers have a high degree of leisure benefits from participating in leisure activities, among which the "psychology benefits" factor $(\mathrm{M}=4.265)$ is the highest, and the 
"social benefits" factor is the lowest score $(\mathrm{M}=3.162)$. The main leisure benefits of elementary schoolteachers in Tainan County for participating in leisure activities are obtaining psychology benefits.

Table 6. Demographic result on leisure benefits

\begin{tabular}{lcc}
\hline & Mean & Std. Deviation \\
\hline Leisure Benefits & 3.887 & 6.485 \\
Physiological Benefits & 4.089 & 2.941 \\
Psychology Benefits & 4.265 & 2.671 \\
Social Benefits & 3.162 & 1.659 \\
\hline
\end{tabular}

\subsubsection{Demographic Analysis of Happiness}

Table 7 shows the demographic analysis result of happiness $(M=3.925)$, indicating that elementary schoolteachers have a high degree of happiness in participating in leisure activities, among which the "interpersonal communication" factor $(M=4.080)$ is the highest and the "health" factor is the lowest score $(\mathrm{M}=3.709)$. The main happiness of elementary schoolteachers in Tainan County in participating in leisure activities is improving interpersonal communication.

Table 7. Demographic results on happiness

\begin{tabular}{lcc}
\hline & Mean & Std. Deviation \\
\hline Happiness & 3.925 & 12.224 \\
Life Satisfaction & 3.930 & 4.202 \\
Interpersonal Communication & 4.080 & 2.867 \\
Assertiveness & 3.944 & 3.392 \\
Health & 3.709 & 3.614 \\
\hline
\end{tabular}

\subsection{Independent Sample t-test Analysis}

Table 8 shows that the "leisure involvement", "leisure benefits" and "happiness" of male teachers in participating leisure activities reach a significant difference compared to female teachers $(\mathrm{t}=34.310, p<.01 ; \mathrm{t}=31.589, p<.01 ; \mathrm{t}=25.780, p<.01$, respectively), which means male elementary schoolteachers in participating leisure activities are affected by the attraction factor $(\mathrm{t}=32.245, p<.01)$ and have higher degree of leisure benefits including physiological benefits, psychology benefits, and social benefits factors, as well as have higher degree of happiness including life satisfaction, interpersonal communication, assertiveness, and health factors. 
3.5 One-Way Anova Analysis

3.5.1 One-Way Anova Analysis of Leisure Involvement

Table 8. Results of independent sample $t$-text statistics

\begin{tabular}{|c|c|c|c|c|c|c|}
\hline & Factor & Item & Mean & $\begin{array}{c}\text { Std. } \\
\text { Deviation }\end{array}$ & $\mathrm{T}$ & Scheff's \\
\hline \multirow{8}{*}{$\begin{array}{c}\text { Leisure } \\
\text { Involvement }\end{array}$} & & Male & 60.137 & 6.670 & \multirow{2}{*}{$34.310^{* *}$} & \multirow{2}{*}{$1>2$} \\
\hline & & Female & 56.205 & 7.107 & & \\
\hline & \multirow{2}{*}{ Attraction } & Male & 23.788 & 1.920 & \multirow{2}{*}{$32.245^{*}$ * } & \multirow{2}{*}{$1>2$} \\
\hline & & Female & 22.504 & 2.515 & & \\
\hline & \multirow{2}{*}{$\begin{array}{l}\text { Centrality of } \\
\text { Lifestyle }\end{array}$} & Male & 19.993 & 3.757 & \multirow{2}{*}{$13.654^{* *}$} & \multirow{2}{*}{$1>2$} \\
\hline & & Female & 18.699 & 3.615 & & \\
\hline & \multirow{2}{*}{$\begin{array}{c}\text { Self- } \\
\text { Expression }\end{array}$} & Male & 16.356 & 2.562 & \multirow{2}{*}{$31.698^{* *}$} & \multirow{2}{*}{$1>2$} \\
\hline & & Female & 15.022 & 2.459 & & \\
\hline \multirow{8}{*}{$\begin{array}{l}\text { Leisure } \\
\text { Benefits }\end{array}$} & \multirow{4}{*}{$\begin{array}{c}\text { Physiological } \\
\text { Benefits }\end{array}$} & Male & 56.952 & 6.716 & \multirow{2}{*}{$31.589^{* *}$} & \multirow{2}{*}{$1>2$} \\
\hline & & Female & 53.546 & 6.175 & & \\
\hline & & Male & 21.740 & 2.875 & \multirow{2}{*}{$40.784^{* *}$} & \multirow{2}{*}{$1>2$} \\
\hline & & Female & 19.998 & 2.833 & & \\
\hline & \multirow{2}{*}{$\begin{array}{l}\text { Psychology } \\
\text { Benefits }\end{array}$} & Male & 21.144 & 2.724 & \multirow{2}{*}{$19.073^{* *}$} & \multirow{2}{*}{$1>2$} \\
\hline & & Female & 21.042 & 2.596 & & \\
\hline & \multirow{2}{*}{$\begin{array}{c}\text { Social } \\
\text { Benefits }\end{array}$} & Male & 13.068 & 1.676 & \multirow{2}{*}{$12.763^{* *}$} & \multirow{2}{*}{$1>2$} \\
\hline & & Female & 12.506 & 1.630 & & \\
\hline \multirow{10}{*}{ Happiness } & & Male & 98.534 & 13.396 & \multirow{2}{*}{$25.780 * *$} & \multirow{2}{*}{$1>2$} \\
\hline & & Female & 92.706 & 11.436 & & \\
\hline & \multirow{2}{*}{ Life Satisfaction } & Male & 28.658 & 4.415 & \multirow{2}{*}{$14.948^{* *}$} & \multirow{2}{*}{$1>2$} \\
\hline & & Female & 27.118 & 4.058 & & \\
\hline & \multirow{2}{*}{$\begin{array}{l}\text { Interpersonal } \\
\text { Communication }\end{array}$} & Male & 25.219 & 3.172 & \multirow{2}{*}{$13.393^{* *}$} & $1>2$ \\
\hline & & Female & 24.224 & 2.712 & & $1>2$ \\
\hline & Assertiveness & Male & 24.685 & 3.529 & $18315^{* *}$ & $1>?$ \\
\hline & RDSECIIVCHCSS & Female & 23.313 & 3.276 & 10.510 & $1>2$ \\
\hline & Health & Male & 19.973 & 3.758 & 32393 * * & $1>2$ \\
\hline & Hedin & Female & 18.052 & 3.432 & 32.393 & $1>2$ \\
\hline
\end{tabular}

${ }^{*} p<.05,{ }^{*}{ }^{*} p<.01,{ }^{* * *} p<.001$. 
Table 9. Summary results of on-way anova on leisure involvement

\begin{tabular}{|c|c|c|c|c|c|c|}
\hline Factor & Demographic & Item & Mean & $\begin{array}{c}\text { Std. } \\
\text { Deviation }\end{array}$ & $\mathrm{F}$ & Scheff's \\
\hline \multirow{4}{*}{ Attraction } & \multirow{4}{*}{ Age } & Under 30 years & 23.519 & 2.303 & \multirow{4}{*}{$3.263^{*}$} & \multirow{4}{*}{$1>2$} \\
\hline & & $31-40$ years old & 22.513 & 2.506 & & \\
\hline & & $41-50$ years old & 22.858 & 2.360 & & \\
\hline & & 51-60 years old & 22.882 & 2.728 & & \\
\hline \multirow{5}{*}{$\begin{array}{l}\text { Self- } \\
\text { Expression }\end{array}$} & \multirow{5}{*}{ Job Position } & Director Teacher & 16.455 & 3.346 & \multirow{5}{*}{$2.934^{*}$} & \multirow{5}{*}{$1>3$} \\
\hline & & $\begin{array}{l}\text { Administrative } \\
\text { Teacher }\end{array}$ & 15.611 & 2.446 & & \\
\hline & & Homeroom & 15185 & 2,471 & & \\
\hline & & Teacher & $15.10 J$ & $2.4 / 1$ & & \\
\hline & & Subject Teacher & 15.333 & 2.585 & & \\
\hline
\end{tabular}

${ }^{*} p<.05,{ }^{*}{ }^{*} p<.01,{ }^{*}{ }^{*}{ }^{*} p<.001$.

Table 9 shows the demographic results of age and job position on "leisure involvement" in participating in leisure activities reaching a significant difference. For the "attraction" aspect indicated that teachers aged below 30 years have a higher degree of significant difference than do teachers aged between 31-40 years $(\mathrm{F}=3.263, p<.05)$; for the "self-expression" aspect, director teachers have a higher degree of significant difference than do homeroom teachers $(\mathrm{F}$ $=2.934, p<.05)$. H1 was supported.

\subsubsection{One-Way Anova of Leisure Benefits}

Table 10. Summary results of one-way anova on leisure benefits

\begin{tabular}{|c|c|c|c|c|c|c|}
\hline Factor & Demographic & Item & Mean & $\begin{array}{c}\text { Std. } \\
\text { Deviation }\end{array}$ & $\mathrm{F}$ & Scheff's \\
\hline \multirow{4}{*}{$\begin{array}{l}\text { Physiological } \\
\text { Benefits }\end{array}$} & \multirow{4}{*}{$\begin{array}{l}\text { Teaching } \\
\text { Years }\end{array}$} & Below10 years & 20.017 & 2.837 & \multirow{4}{*}{$5.053 * *$} & \multirow{4}{*}{$\begin{array}{l}3>1 \\
3>2\end{array}$} \\
\hline & & $11-20$ years & 20.288 & 3.103 & & \\
\hline & & 21-30 years & 21.184 & 2.692 & & \\
\hline & & Above 31 years & 19.778 & 3.032 & & \\
\hline \multirow{5}{*}{$\begin{array}{l}\text { Social } \\
\text { Benefits }\end{array}$} & \multirow{5}{*}{ Job Position } & Director Teacher & 13.303 & 1.551 & \multirow{5}{*}{$3.125^{*}$} & \multirow{5}{*}{$1>3$} \\
\hline & & Administrative & 12.874 & 1.545 & & \\
\hline & & $\begin{array}{l}\text { leacher } \\
\text { Homeroom }\end{array}$ & & & & \\
\hline & & Teacher & 12.519 & 1.682 & & \\
\hline & & Subject Teacher & 12.720 & 1.657 & & \\
\hline
\end{tabular}


Table 10 shows the demographic results of teaching years and job position on "leisure benefits" in participating in leisure activities that reach a significant difference. Teachers with 21-30 teaching years have a higher degree of significant difference than do teachers with fewer than 20 teaching years on the "physiological benefits" factor $(\mathrm{F}=5.053, p<.01)$. Teachers who are director teachers also have a higher degree of significant difference than do homeroom teachers on the "social benefits" factor $(\mathrm{F}=3.125, p<.05)$. H2 was supported.

3.5.3 One-Way Anova Analysis of Happiness

Table 11. One-way anova on happiness

\begin{tabular}{|c|c|c|c|c|c|c|}
\hline Factor & Demographic & Item & Mean & $\begin{array}{c}\text { Std. } \\
\text { Deviation }\end{array}$ & $\mathrm{F}$ & Scheff's \\
\hline \multirow{9}{*}{ Life Satisfaction } & \multirow{4}{*}{$\begin{array}{l}\text { Teaching } \\
\text { Years }\end{array}$} & Below 10 years & 26.848 & 3.991 & \multirow{4}{*}{$2.657^{*}$} & \multirow{4}{*}{$3>1$} \\
\hline & & $11-20$ years & 27.615 & 4.259 & & \\
\hline & & $21-30$ years & 28.120 & 4.313 & & \\
\hline & & Above 31 years & 27.333 & 3.640 & & \\
\hline & \multirow{5}{*}{ Job Position } & Director Teacher & 3.830 & 3.328 & \multirow{5}{*}{$4.007^{*}$} & \multirow{5}{*}{$\begin{array}{l}1>3 \\
1>4\end{array}$} \\
\hline & & Administrative & 3.595 & 2.885 & & \\
\hline & & Homeroom & & & & \\
\hline & & Teacher & 4.191 & 2.751 & & \\
\hline & & Subject Teacher & 4.831 & 6.035 & & \\
\hline \multirow{8}{*}{$\begin{array}{l}\text { Interpersonal } \\
\text { Communication }\end{array}$} & \multirow{3}{*}{$\begin{array}{l}\text { Education } \\
\text { Degree }\end{array}$} & College & 22.455 & 3.328 & \multirow{3}{*}{$3.712^{*}$} & \multirow{3}{*}{$3>1$} \\
\hline & & University & 24.409 & 2.885 & & \\
\hline & & Master Above & 24.756 & 2.751 & & \\
\hline & \multirow{5}{*}{ Job Position } & Director Teacher & 3.830 & 3.328 & \multirow{5}{*}{$4.117^{* *}$} & \multirow{5}{*}{$\begin{array}{l}1>3 \\
1>4\end{array}$} \\
\hline & & Administrative & 3.595 & 2.885 & & \\
\hline & & Teacher & & & & \\
\hline & & Teacher & 4.191 & 2.751 & & \\
\hline & & Subject Teacher & 4.831 & 6.035 & & \\
\hline \multirow{4}{*}{ Health } & \multirow{4}{*}{$\begin{array}{l}\text { Teaching } \\
\text { Years }\end{array}$} & Below 10 years & 17.775 & 3.449 & \multirow{4}{*}{$6.655^{*}$} & \multirow{4}{*}{$3>1$} \\
\hline & & $11-20$ years & 18.513 & 3.877 & & \\
\hline & & 21-30 years & 19.494 & 3.192 & & \\
\hline & & 31 years above & 17.778 & 3.598 & & \\
\hline
\end{tabular}

${ }^{*} p<.05,{ }^{*}{ }^{*} p<.01,{ }^{*}{ }^{*}{ }^{*} p<.001$. 
Table 11 shows the demographic results of teaching years, job position, and education degree on "happiness" in participating in leisure activities reaching a significant difference. Teachers with 21-30 teaching years have a higher degree of significant difference than those with under 10 teaching years $(\mathrm{F}=2.657, p<.05)$, and director teachers have a higher degree of significant difference than do homeroom teachers and subject teachers $(\mathrm{F}=4.007, p<.05)$ on the "life satisfaction" factor. For the interpersonal communication aspect, teachers with a master's degree in education have a higher degree of significant difference compared to college education teachers $(\mathrm{F}=3.712, p<.05)$, and director teachers have a higher degree of significant difference than do homeroom teachers and subject teachers $(\mathrm{F}=4.117, p<.01)$. In the health aspect, teachers with 21-30 teaching years have a higher degree of significant difference than teachers with under 10 teaching years $(\mathrm{F}=6.655, p<.05)$. $\mathrm{H} 3$ was supported.

\subsection{Correlation Analysis}

\subsubsection{Relationship between Leisure Involvement and Leisure Benefits}

Table 12 shows a summary of the correlation statistics results. We found that leisure involvement and leisure benefits reached statistical significance $\left(R^{2}=.666, p<.01\right)$, regardless of the effect of any leisure involvement factor on each leisure benefits factor. Both the attraction and centrality of lifestyle factors of leisure involvement have a positive relationship with the psychology benefits factor of leisure benefits $\left(R^{2}=.524, p<.01 ; R^{2}\right.$ $=.501, p<.01$, respectively); the "self-expression" factor of leisure involvement has a positive relationship with the physiological benefits factor of leisure benefits $\left(R^{2}=.573, p\right.$ $<.01$ ). Therefore, greater leisure involvement in participating in leisure activities results in higher leisure benefits; both share a positive relationship. H4 was supported.

Table 12. Correlation analysis results of leisure involvement on leisure benefits

\begin{tabular}{lcllll}
\hline & & $\begin{array}{l}\text { Physiological } \\
\text { Benefits }\end{array}$ & $\begin{array}{l}\text { Psychology } \\
\text { Benefits }\end{array}$ & $\begin{array}{l}\text { Social } \\
\text { Benefits }\end{array}$ & $\begin{array}{l}\text { Leisure } \\
\text { Benefits }\end{array}$ \\
\hline $\begin{array}{l}\text { Leisure } \\
\text { Involvement }\end{array}$ & $R^{2}$ & & & $.666^{* *}$ \\
Attraction & $R^{2}$ & $.441^{* *}$ & $.524^{* *}$ & $.428^{* *}$ & \\
$\begin{array}{l}\text { Centrality of } \\
\text { Lifestyle }\end{array}$ & $R^{2}$ & $.433^{* *}$ & $.501^{* *}$ & $.457^{* *}$ & \\
Self-Expression & $R^{2}$ & $.573^{* *}$ & $.556^{* *}$ & $.538^{* *}$ & \\
\hline
\end{tabular}

${ }^{*} p<.05,{ }^{*}{ }^{*} p<.01,{ }^{*}{ }^{*}{ }^{*} p<.001$.

\subsubsection{Relationship between Leisure Involvement and Happiness}

Table 13 shows the correlation statistics results of leisure involvement and happiness with values reaching statistical significance, $\left(R^{2}=.563, p<.001\right)$; Both the attraction and self-expression factors of leisure involvement have a positive relationship with the interpersonal communication factor of happiness $\left(R^{2}=.329, p<.01 ; R^{2}=.494, p<.01\right.$, 
respectively); the centrality of lifestyle factor of leisure involvement has a positive relationship with the life satisfaction factor of happiness $\left(R^{2}=.472, p<.01\right)$. Therefore, greater leisure involvement in participating in leisure activities results in higher happiness; both share a positive relationship. H5 was supported.

Table 13 Correlation analysis results of leisure involvement on happiness

\begin{tabular}{lccllll}
\hline & \multicolumn{2}{c}{$\begin{array}{l}\text { Life } \\
\text { Satisfaction }\end{array}$} & $\begin{array}{l}\text { Interpersonal } \\
\text { Communication }\end{array}$ & Assertiveness & Health & Happiness \\
\hline $\begin{array}{l}\text { Leisure } \\
\text { Involvement }\end{array}$ & $R^{2}$ & & & & $.563^{* * *}$ \\
Attraction & $R^{2}$ & $.278^{* *}$ & $.329^{* *}$ & $.247^{* *}$ & $.237^{* *}$ & \\
$\begin{array}{l}\text { Centrality of } \\
\text { Lifestyle }\end{array}$ & $R^{2}$ & $.472^{* *}$ & $.461^{* *}$ & $.468^{* *}$ & $.466^{* *}$ & \\
Self-Expression & $R^{2}$ & $.435^{* *}$ & $.494^{* *}$ & $.447^{* *}$ & $.418^{* *}$ & \\
\hline
\end{tabular}
${ }^{*} p<.05,{ }^{*}{ }^{*} p<.01,{ }^{*}{ }^{*}{ }^{*} p<.001$.

\subsubsection{Relationship between Leisure Benefits and Happiness}

Table 14 shows the correlation statistics results of leisure benefits and happiness with values reaching statistical significance $\left(R^{2}=.516, p<.01\right)$. Physiological benefits, psychology benefits, and social benefits have a positive relationship with interpersonal communication $\left(R^{2}=.438, p<.01 ; R^{2}=.467, p<.01 ; R^{2}=.488, p<.01\right.$, respectively). Therefore, greater leisure benefits in participating in leisure activities results in higher happiness; both share a positive relationship. H6 was supported.

Table 14. Correlation analysis results of leisure benefits on happiness

\begin{tabular}{|c|c|c|c|c|c|c|}
\hline & & $\begin{array}{l}\text { Life } \\
\text { Satisfaction }\end{array}$ & $\begin{array}{l}\text { Interpersonal } \\
\text { Communication }\end{array}$ & Assertiveness & Health & Happiness \\
\hline $\begin{array}{l}\text { Leisure } \\
\text { Benefits }\end{array}$ & $R^{2}$ & & & & & $.516^{* *}$ \\
\hline $\begin{array}{l}\text { Physiological } \\
\text { Benefits }\end{array}$ & $R^{2}$ & $.408^{* *}$ & $.438^{* *}$ & $.392^{* *}$ & $.380^{* *}$ & \\
\hline $\begin{array}{l}\text { Psychology } \\
\text { Benefits }\end{array}$ & $R^{2}$ & $.387^{* *}$ & $.467^{* *}$ & $.380^{* *}$ & $.354^{* *}$ & \\
\hline Social Benefits & $R^{2}$ & $.424^{* *}$ & $.488^{* *}$ & $.412^{* *}$ & $.304^{* *}$ & \\
\hline
\end{tabular}

${ }^{*} p<.05,{ }^{*}{ }^{*} p<.01, * * * p<.001$. 


\subsection{Regression Analysis}

Table 15 shows the effect of centrality of lifestyle and self-expression of leisure involvement on happiness yields meaningful results. The centrality of lifestyle factor of leisure involvement can positively predicts happiness, including factors of life satisfaction, interpersonal communication, assertiveness and health. $(\mathrm{F}=.295, p<.01 ; \mathrm{F}=.177, p<.01, \mathrm{~F}$ $=.293, p<.01 ; \mathrm{F}=.335, p<.01$, respectively). Therefore, happiness of participating in leisure activities and leisure involvement are positively related. This means that a higher leisure involvement of participating leisure activities results in greater happiness. The effect of leisure benefits on happiness also yields meaningful results with statistical significance ( $\mathrm{F}$ $=.370, p<.01$ ), which means that leisure benefits and happiness have a significant regression relationship. The results show that leisure involvement and leisure benefits accounted for $38.7 \%$ of the variance in happiness. $\mathrm{H} 7$ was supported.

Table 15. Summary of regression analysis

\begin{tabular}{|c|c|c|c|c|c|}
\hline & $\begin{array}{l}\text { Life } \\
\text { Satisfaction }\end{array}$ & $\begin{array}{l}\text { Interpersonal } \\
\text { Communication }\end{array}$ & Assertiveness & Health & Happiness \\
\hline $\begin{array}{l}\text { Leisure } \\
\text { Involvement }\end{array}$ & & & & & .002 \\
\hline Attraction & -.034 & .004 & -.074 & -.064 & -.050 \\
\hline $\begin{array}{l}\text { Centrality } \\
\text { Of Lifestyle }\end{array}$ & $.295^{* *}$ & $.177^{* *}$ & $.293^{* *}$ & $.335^{* *}$ & $.323^{* *}$ \\
\hline $\begin{array}{l}\text { Self- } \\
\text { Expression }\end{array}$ & .093 & $.181^{* *}$ & $.133^{* *}$ & $.122^{* *}$ & $.147^{* *}$ \\
\hline $\begin{array}{l}\text { Leisure } \\
\text { Benefits }\end{array}$ & & & & & $.370^{* * *}$ \\
\hline $\begin{array}{l}\text { Physiological } \\
\text { Benefits }\end{array}$ & .015 & .008 & .003 & .013 & .011 \\
\hline $\begin{array}{l}\text { Psychology } \\
\text { Benefits }\end{array}$ & $-.199^{*}$ & .018 & -.143 & -.158 & -.151 \\
\hline $\begin{array}{l}\text { Social } \\
\text { Benefits }\end{array}$ & .090 & $.174^{* *}$ & .093 & -.109 & .066 \\
\hline$R$ & .548 & .587 & .543 & .516 & .622 \\
\hline$R^{2}$ & .300 & .345 & .295 & .266 & .387 \\
\hline
\end{tabular}

\section{Discussion}

The attraction factor is the main consideration of leisure involvement for elementary schoolteachers participating in leisure activities. A greater attractiveness of leisure activities and higher leisure involvement means greater attention has been directed toward conceptualizing and measuring leisure involvement (Havitz et al., 1993; McIntyre, 1989; 
Schuett, 1993). The result of the independent sample $t$-test indicated that male elementary schoolteachers have a higher significant difference than do female teachers on leisure involvement. The finding is in line with previous studies (Robinson \& Godbey, 1993; Statistics Canada, 1994) that indicates the men are considerably more likely than women to participate in organized and informal sports activities. Owning to most stereotyping sports and leisure activities are considered to be masculine (Metheny, 1967); thus, male elementary teachers have more physical force to participate in leisure activities than do female elementary teachers.

The One-Way ANOVA results also indicated a significant difference on the effect of age on leisure involvement, particularly for teachers under 30 years of age. This finding is compatible with previous studies (Alasheev \& Bykov, 2002; Chen, 2004). Owing to the heavy administration work, teaching pressure, family factors, mental and physiological energy are too more need to take for experienced and older teachers (who are over 30 years old) to do than younger teachers. On account of these factors increase social anxiety, older teachers experience greater difficulty arranging and planning leisure activities; thus, younger teachers have more time and energy for leisure involvement in participating in leisure activities.

Regardless of the type of leisure activities, more involvement leads to greater life satisfaction (Tsai et al., 2001). Rejeski et al. (2001) studied people who are sedentary and found that increasing physical activities has a positive relationship on a sense of well-being and satisfactory bodily functions. Thogersen-Ntouman et al. (2005) indicated that exercise had a direct and indirect influence on the happiness of technology company employees. Lu and $\mathrm{Hu}$ (2005) found that leisure involvement had a positive correlation with leisure satisfaction and happiness among college students, particularly involvement in hobbies, sports, and indoor activities. Therefore, continuous involvement in particular leisure activities relieves life pressures and yields a higher degree of psychological well-being (Reich \& Zautra, 1981).

The One-Way ANOVA results indicated that "teaching years" and "job position" both have a significant effect on leisure benefits. Teachers with 11-20 teaching years show a significant difference on physiological and psychological benefits compared to teachers with under 11 teaching years. This finding is consistent with those by Nakamura et al. (1990), Lin and Gina (1995) and McGuire et al. (2004). May be experienced and older teachers, who are more attention on the physiological and psychological benefits of leisure activities, including relieving stress, improving health, and slowing aging, thus they will focus more concerned regarding health to well arrange whose leisure time recreation. Director teachers who take on the administration work significantly differ from homeroom teachers on the social benefits of participating in leisure activities, because in addition to teaching, director teachers who assume administration duties require a greater capacity for supervision, management, and coordination. Participating in leisure activities helps reduce interpersonal conflict.

The results of One-Way ANOVA on happiness indicated that elementary schoolteachers participate in leisure activities to obtain happiness from interpersonal communication, because participating in leisure activities helps people make friends and share with friends, 
and provides social benefits from social support and companionship, which is the most important interpersonal relationship factor. Teachers with 21-30 teaching years are more concerned regarding life satisfaction and mental and physiological health than teachers with under 10 teaching years; and teachers with a master's education degree are more concerned regarding the interpersonal communication factor of participating in leisure activities. Which is compatible with previous studies (Pagano et al., 2006; Chen, 2006; Gu, 2006; Hou, 2006). Because of experienced and older teachers emphasize the effect of happiness on participating in leisure activities that can provide mental health benefits and enhance happiness. Teachers with a higher educational background compared to general university-graduated teachers have a better chance to handle administration work. Therefore, a greater emphasis on interpersonal communication leads to efficient management and coordination of administrative affairs and interpersonal happiness.

The correlation analysis results indicated that the attraction factor of leisure involvement is positively related to the psychology factor of leisure benefits and the interpersonal communication factor of happiness. These results are consistent with previous studies (Lu \& Argyle, 1994; Tinsley \& Tinsley, 1986; Fun, 1997; Kujala et al., 1998; Currier, 2004) that reported that leisure involvement has a positive effect including relaxation, stress, anxiety, confidence, good interpersonal relationships, and self-accomplishment. This means that elementary schoolteachers prefer participating in interesting and attractive leisure activities, and participating in leisure activities with friends enhances interpersonal relationships and obtains the highest degree of happiness (Csikszentmihalyi \& Hunter, 2003) and relaxation.

Finally, the regression results show that leisure involvement and leisure benefits positively predict happiness and have a direct influence on happiness, the finding is consistent with previous studies (Berger \& McInman, 1993; Lin, 2004), including participating in leisure activities fosters positive emotions (Argyle \& Corssland, 1987; Hills \& Argyle, 1998), promotes trust and support from friends (Wu, 2006), and provides physical mental happiness. Csikszentmihalyi and Hunter (2003) indicated that people who participate in passive leisure activities are associated with different degrees of happiness. Thus, a higher degree of leisure involvement and leisure benefits yields a higher degree of happiness.

\section{Conclusion and Recommendation}

\subsection{Conclusion}

We examined the relationship among leisure involvement, leisure benefits, and happiness, and the results indicate that elementary schoolteachers in Tainan County have positive feelings on different factors of leisure involvement, leisure benefits, and happiness. All of the factors have a significant positive relationship. Leisure involvement and leisure benefits positively predict respondent happiness.

\subsection{Recommendation}

The findings indicate that a significant difference between gender and job position on leisure involvement, leisure benefits, happiness and a higher leisure involvement of elementary schoolteachers yields greater happiness. Therefore, the contribution of this paper is we can 
suggest that elementary schoolteachers need to participate in more leisure activities and encourage they integrate leisure habits into their life to contribute to their physical and mental health and happiness, especially for female teachers and homeroom teachers; moreover, schools can conduct a leisure activities competition between teachers to provide teachers with more opportunities for leisure activities, and to enhance motivation to participate. We also can suggest the Department of Education design some interesting and attractive leisure activities in summer or winter vacation to encourage elementary schoolteachers to participate, then teaching more leisure value to students.

\section{Reference}

Alasheev, S. L., \& Bykov, S. V. (2002). Teachers state of Anxiety. Russian Education and Society, 44(12), 62-72. http://dx.doi.org/10.2753/RES1060-9393441262

Argyle, M. (1987). The psychology of happiness. USA : Methuen: Londen.

Argyle, M., \& Crossland, J. (1987). The dimensions of positive emotions. British Journal of Social Psychology, 26, 127-137. http://dx.doi.org/10.1111/j.2044-8309.1987.tb00773.x

Ateca-Amestoy, V., Serrano-del-Rosal, R., \& Vera-Toscano, E. (2008). The leisure experience. The Journal of Socio-Economics, 37(1), 64-78. http://dx.doi.org.ezproxy.libdb.yuntech.edu.tw:2048/10.1016/j.socec.2006.12.025

Berger, B. C., \& McInman. (1993). Exercise and the quality of life. In R. N. M. Singer, L. K. Murpuh, \& Tennant (Eds.). Handbook of research on sport psychology (pp. 729-760). New York: Macmillan Publishing.

Berger, B. G., \& Wankel, L. M. (1991). The personal and social benefits of sport and physical activity. In B. L. Driver, P. J. Brown, \& G. L. Peterson (Eds.), Benefits of leisure (pp. 121-144).

Chang, H. W., Xie, Z. W., \& Wang, J. J. (2001). The study of recreational sports participation and impediments factors of university staff - The example of $\mathrm{Fu}$ Jen University. Taiwan University of Arts, 68, 121-134.

Chen, T. D. (2006). The comparative study of social support and happiness with regular classes and special education junior teachers. Unpublished Master's Thesis, National Kaohsiung Normal University, Kaohsiung.

Chen, Y. P. (2004). The study of factors of happiness with Elementary schoolteachers. Unpublished Master's Thesis, National Pingtung University of Education, Pingtung.

Cordes, K. A., \& Ibrahim, H. M. (1999). Applications in recreation \& leisure for today and the future. Boston : McGraw-Hill.

Csikszentmihalyi, M., \& Hunter, J. (2003). Happiness in Everyday Life: The uses of Experience Sampling. Journal of Happiness Studies, 4(2), 185-199. http://dx.doi.org/10.1023/A:1024409732742 
Currier, J. (2004). Motherhood, stress and the exercise experience: freedom or constraint? Leisure Studies, 23(3), 225-242. http://dx.doi.org/10.1080/0261436042000251987

Damanedier, J. (1974). Sociology of leisure. New York : Elsevier North-Holland.

Dattilo, J., \& Murphy, W. (1991). Leisure education program planning: A systematic approach. State College, PA: Venture Publishing Inc.

Diener, E. (1984). Subjective well-being. Psychological Bulletin, 95(3), 542-575. http://dx.doi.org/10.1037/0033-2909.95.3.542

Diener, E., \& Seligman, M. (2002). Very happy people. Psychological Science, 13, 81-84. http://dx.doi.org/10.1111/1467-9280.00415

Fun, J. L. (1997). Principles and Labs for Fitness and Wellness. Taipei: Han-Wen.

Godbey, G. (1999). Leisure in your life: an exploration. State College, PA: Venture publishing, Inc.

Gu, T. G. (2006). The study of happiness and related factors of Junior high school teachers. Unpublished Master's Thesis, National Taiwan Normal University, Taipei.

Hallab, Z. (2006). Catering to the healthy-living vacationer. Journal of Vacation Marketing, 12(1), 71-91. http://dx.doi.org/10.1177/1356766706059043

Havitz, M. E., \& Dimanche, F. (1997). Leisure involvement revisited: Conceptual conundrums and measurement advances. Journal of Leisure Research, 29(3), 245 -278.

Havitz, M. E., Green, T. R., \& McCarville, R. E. (1993). Order effects and the measurement of enduring leisure involvement. Journal of Applied Recreation Research, 18, 181-195.

Hills, P., \& Argyle, M. (1998). Positive moods derived from leisure and their relationship to happiness and personality. Personality and Individual Differences, 25, 523-535. http://dx.doi.org.ezproxy.libdb.yuntech.edu.tw:2048/10.1016/S0191-8869(98)00082-8

Hong, W. C. (2002). The study of leisure activities after school and holiday. Journal of Sports, $68,101-106$.

Hou, C. Y. (2006). The study of happiness and teaching effectiveness of elementary schoolteachers. Master's Thesis, Chung Yuan Christian University. Chung Li.

Huang,Y. L., \& Chen, H. M. (2005). The study of leisure attitude and behavior. Journal of Outdoor Recreation, 18(3), 81-102.

Kaiser, H. F. (1974). Little Jiffy, Mark Iv. Educational and Psychological Measurement, 34(1), 111-117. http://dx.doi.org/10.1177/001316447403400115

Kraus, R. G. (1994). Tomorrow's leisure: Meeting the challenges. Journal of Physical Education, Recreation \& Dance, $\quad$ 65(4), http://dx.doi.org/10.1080/07303084.1994.10606897 
Kujala, U. M., Kaprio, J., Sarna, S., \& Koskenvuo, M. (1998). Relationship of leisure-time physical activity and mortality: The Finnish twin cohort. Journal of the American medical Association, 279(6), 440-444. http://dx.doi.org/10.1001/jama.279.6.440

Lin, H. H. (2002). The effect of the interpretive experience of leisure benefits - as an example of hikers. Unpublished Master's Thesis, National Taiwan Normal University, Taipei.

Lin, N., \& Gina, L. (1995). Urban stress in China. Social Science and Medicine, 41(8),1131-1145. http://dx.doi.org/10.1016/0277-9536(94)00426-T

Lin, Y. M. (2004). The study of the leisure benefit and happiness of swimmers. Unpublished Master's Thesis, National Yunlin University of Science and Technology. Yunlin.

Lu, L., \& Argyle, M. (1994). Leisure satisfaction and happiness as a function of leisure activity. Kaohsiung Journal Medicine Science, 10, 89-96.

Lu, L., \& Hu, C. H. (2005). Personality, leisure experiences and happiness. Journal of Happiness studies, 6(3), 325-342. http://dx.doi.org/10.1007/s10902-005-8628-3

McGuire, F. A., Boyd, R. K., \& Tedrick, R. E. (2004). Leisure and Aging: Ulyssean Living in Later Life. Champaign. IL: Sagamore Publisher.

McIntyre, N. (1989). The personal meaning participation: Enduring involvement. Journal of Leisure Research, 21(2), 167-179.

McIntyre, N., \& Pigram, J. J. (1992). Recreation specialization reexamined: The case of vehicle-based campers. Leisure Sciences, $14(1), \quad 3-15$. http://dx.doi.org/10.1080/01490409209513153

Metheny, E. (1967). Connotations of movement in sport and dance. Dubuque, IA: William, C. Brown.

Nakamura, E., Moritani, T., \& Kanetaka, A. (1990). Biological age versus physical fitness age. European Journal of Applied Physical, 61, 202-208. http://dx.doi.org/10.1007/BF00357600

Nawijn, J. (2011). Happiness Through Vacationing: Just a Temporary Boost or Long-Term Benefits? Journal of Happiness Studies, $12, \quad 651-665$. http://dx.doi.org/10.1007/s10902-010-9221-y

Nunnally, J. C. (1978). Psychometric Theory (2nd ed.). New York: McGraw-Hill.

Pagano, Barkhoff, Heiby, \& Schlicht. (2006). Dynamical modeling of the relations between leisure activities and health indicators. Journal of Leisure Research, 38(1), 61-77.

Pichly, A. (2002). Individuals can benefit physically, mentally, emotionally, spiritually and socially from a comprehensive leisure education program implemented in the schools during childhood. A research project report submitted in partial fulfillment of the requirements for the successful completion of RLS 209.

Reich, J. W. \& Zautra, A. (1981). Life events and personal causation: some relationships with 
satisfaction and distress. Journal of Personality and Social Psychology, 41, 1002-1012. http://dx.doi.org/10.1037/0022-3514.41.5.1002

Rejeski, W. J., Shelton, B., Miller, M., Dunn, A. L., King, A. C., \& Sallis, J. F. (2001). Mediators of increased physical activity and change in subjective well-being: Results from the Activity Counseling Trial (ACT). Journal of Health Psychology, 6(2), 159-168. http://dx.doi.org/10.1177/135910530100600206

Robinson, J. P., \& Godbey, G. (1993). Sport, fitness and the gender gap. Leisure Science, 15(4).291-307. http://dx.doi.org/10.1080/01490409309513208

Rothschild, M. L. (1984). Perspectives on involvement: Current problems and future directions. Advances in Consumer Research, 11, 216-217.

Schuett, M. A. (1993). Refining measures of adventure recreation involvement. Leisure Sciences, 15(3), 205-216. http://dx.doi.org/10.1080/01490409309513200

Sheldon, K. M., \& Lyubomirksy, S. (2006). Achieving sustainable gains in happiness: Change your actions, not your circumstances. Journal of Happiness Studies, 7(1), 55-86. http://dx.doi.org/10.1007/s10902-005-0868-8

Sherif, C. W., Sherif, M., \& Nebergall, R. E. (1965). Attitude and attitude change: The social judgment involvement approach. Philadelphia: WB Saunders.

Shivers, J. S. (1981). Leisure and Recreation Concepts: A Critical Analysis. Boston: Allyn \& Bacon, Inc.

Sparks, B., Bowen, J., \& Klag, S. (2003). Restaurants and the tourist market. International Journal of Contemporary Hospitality Management, 15(1), 6-13. http://dx.doi.org/10.1108/09596110310458936

Spiers, A., \& Walker, G. J. (2008). The effects of ethnicity and leisure satisfaction on happiness, peacefulness, and quality of life. Leisure Sciences, 31(1), 84-99. http://dx.doi.org/10.1080/01490400802558277

Statistics Canada. (1994). Sport participation in Canada. Ottawa, ON: Ministry of Supply and Services.

Thogersen-Ntouman, C., Fox, K. R., \& Ntoumanis, N. (2005). Relationships between exercise and three components of mental well-being in corporate employee. Psychology of Sport and Exercise, 6, 609-627. http://dx.doi.org/10.1016/j.psychsport.2004.12.004

Tinsley, H. E. A., \& Tinsley, D. J. (1986). A theory of the attributes, benefits and causes of leisure experience. Leisure Sciences, 8, 1-45. http://dx.doi.org/10.1080/01490408609513056

Tsai, A. Q., Liu, X. X., \& Huang, S. Z. (2001). The relationship of leisure participation and life satisfaction of retirement elderly people. Unpublished Master's Thesis. Journal of National Kaohsiung University of Applied Science, 31, 183-221.

Tsai, C. E. (2004). The study of job stress, corresponding way and job satisfaction of Taipei 


\section{Macrothink}

International Research in Education

ISSN 2327-5499 2013, Vol. 1, No. 1

Elementary Schoolteachers. Unpublished Master's Thesis, National Taipei University of Education. Taipei.

Wu, M. L., \& Tu, J. T. (2005). Spss \& the application and analysis of statistics. Taipei: Wu-Nan.

Wu, Y. F. (2006). The study of sustained involvement, leisure benefits and happiness of road race participants. Unpublished Master's Thesis, National Taiwan Sport University, Taoyuan.

\section{Copyright Disclaimer}

Copyright reserved by the author(s).

This article is an open-access article distributed under the terms and conditions of the Creative Commons Attribution license (http://creativecommons.org/licenses/by/3.0/). 